\title{
Beyond Pure Frequency and Phases Exploiting: Color Influence in SSVEP Based on $\mathrm{BCl}$
}

\author{
Mustafa Aljshamee, Mahdi Q. Mohammed, Riaz-Ul-Ahsan Choudhury, Abbas Malekpour and Peter Luksch \\ Department of Computer Science, University of Rostock, Rostock 18059, Germany
}

\begin{abstract}
A BCI (brain computer interface) established a new direct communication channel using the brain activity between the human brain and machine. The visual stimulus with a certain frequency is present to the BCI users; it exists in a particular condition to observe a continuous brain response respect to frequent of visual stimuli. A significant problem when engaged the SSVEP (steady-state visual evoked potential) based on BCI, it will be exhausted and may suffer for the users when staring at flashing stimuli. This experimental study investigates how the differences in LED's-colors influence of SSVEP with respect to (i.e., frequencies and phases). The result shows that the visualization of phase delays in lower frequencies greater than in higher frequencies.
\end{abstract}

Key words: SSVEP, BCI, EEG (electroencephalograms), FFT (fast fourier transform), ERP (event related potential), FPGA (field programmable gate array), LED (light emitting diode).

\section{Introduction}

The BCI (brain computer interfaces) is a new technology to establish a communication channel in between human brain and outside world. The user's pay attention on stimulating, the commands are expressed to the external world based on brain activity.

The most of BCI systems based on SSVEP (steady-state visual evoked potentials) depended on number of stimuli flashing with differences in frequencies or phases, which in turn to direct affectively on occipital region of the brain [1].

Researches in BCI have identified that SSVEP based BCI needs less training time, but provides faster response and higher information transfer rate [2], [3] and [4]; hence it is suitable for implementing real time applications. The observation of rhythmic of visual stimuli gives growing oscillatory on EEG (electroencephalographic) signals with a major frequency component on the frequency of the flashing

Corresponding author: Mustafa Aljshamee, M.Sc., research fields: adaptive signal processing in reconfigurable software / hardware. E-mail: mustafa.aljshamee@uni-rostock.de. stimuli with several harmonics [5].

The SSVEP based BCIs typically involve to use a number of repetitive visual stimuli. This visual stimuli in computer will be translated these stimulating to the specific computer command's or machine instruction by paying attention on one of the visual targets [5].

Often SSVEP signals are corrupted with other noise such as interpretation of EEG data, artifacts, external noise like power-line interference and D.C component. Those noisy signals require efficient strategies for noise prevention using a specific signal processing technique to reduce these undesired effects [6]. However, the common approach in BCI system is involved with a lower frequency stimuli, but in general this will strained users behavioral than a high frequency stimuli [5]. Further a bounding of obvious frequencies that can be limitation in SSVEP BCI systems [7].

The favorite performance typically in analysis and classification on a short chunk of data, that will be reducing the number of time recording, also will reduce the spectral resolution analysis; another factor that can restrict the number of frequencies available is the refresh rate of monitors when such devices are 
used to display as visual stimuli [7]. The color of the visual stimuli is also known to affect the elicited SSVEP paradigms, and a number of BCI studies have investigated which colors give raise strongest SSVEP [5].

Another factor that influences the characteristics of the evoked SSVEPs is the correlation between the stimulus color and the amplitude as well as the topography of the elicited SSVEP components that has been reported in a number of studies [8] and [9].

In the context of BCIs, a number of research groups have investigated which stimulus colors give rise to the strongest SSVEPs [10] and [11]. Nonetheless, of these studies are considering to use a different colors in order to extend the number of choices available in an SSVEP based BCI setup.

A discernment of light for human eye is through the retina which contains two kinds of light-sensitive cells rod and cone cells [11], the rod cells have response on luminance but cannot distinguish different colors [11]. The ability of the human eye to distinguish colors is based upon the varying sensitivity of cone cells to the light of different wavelengths.

Therefore, human eyes will have different discernment to different colors; as a result, where the different colors of the stimuli will elicit different SSVEPs. At present, green, red, gray, black and white stimuli have been used for SSVEP based BCIs [11].

The motivation of this experimental studies as practical BCI system, of multi-colors sequence used to stimulus are driven which can be produced by controlling signal generators.

Implementing with FPGA (field programmable gate array) with aspect to investigated an offline analysis of SSVEP-based on BCI system. Indeed, we conclude two kinds of results using ERP and FFT to find out the phase shifting and power amplitude.

The visual stimulator plays an important role, which presented using flashing with three colors LED's rows, taking in the account the effect of stimulation parameter relying on color stimuli is compared to that of a system where the number of choices available with respect to different phases extended by introducing multi-colors targets. The details of the experimental protocol as well as the methods employed for the analysis are next described.

\section{Materials and Methods}

\subsection{System Configuration}

To inspect the different colors stimuli effects in $\mathrm{BCI}$ systems respect to flecking-LED, indeed utilized a prototype implemented an SSVEP based on BCI control systems. In Fig. 1 illustrates, the main block diagram proposed as low-cost SSVEP based on BCI as close-loop control system.

In general, the multi-stimulus panel prototype obtainable in LED's flickering will be presented in externally stimuli board, which are induced the regular-flicker LED's with phase-tagged toward distinguish brain response.

The flicker frequency is configured only with two frequencies- $6 \mathrm{~Hz}$ and $12 \mathrm{~Hz}$. The stimulus panel will be horizontally fixed on eye level of participants with distance around $80 \mathrm{~cm}$ in between. The multi-stimulus panel of SSVEP as close-loop system will be responded by connecting the FPGA depth board crossing the computer in order to send commands to change individual colors, position and patterns of each LED's on the stimuli board.

To evaluate this experiment, well set a three LED's colors (red, blue and white), as (4 LED's 3 colors) within a rows are distributed in surround and centre together with distance in between for each row-group.

However, the promotion of control on stimuli LED's of prominent controller commands through serial data bus of computer, according to experiments setup conditions.

Utilizing the FPGA core connected to personal computer via serial cable, where the Altera FPGA Cyclone IV EP4CE115F29C7N connected to the stimuli LED's board. The complete hardware design stuff of visual board was powered by pure direct current 


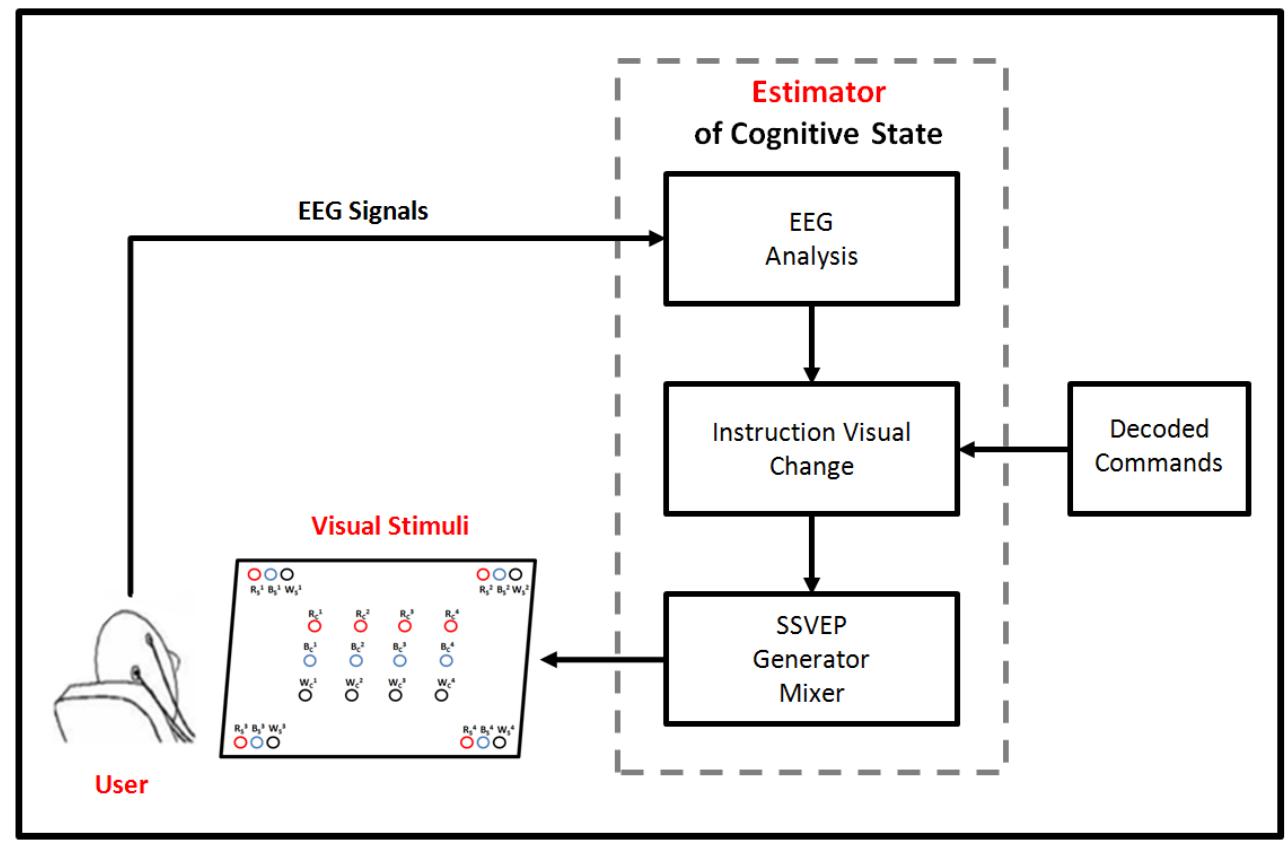

Fig. 1 The main block diagram proposed multi-colors LED's stimuli as a low-cost SSVEP based BCI, a stimuli panel content 24 stimulation position, distributed between surround and center, using promote commands to control in each of positions and colors of LED's respect to experiment protocol.

(DC) power supply of $9 \mathrm{~V}$ battery source to avoid AC (alternating current) interference component.

The multi-stimulus panel includes of 24 visual stimuli position with three different colors distributed between the center and surround assort in rows and corners. Being in experiments associated to find-out the influence of brain responses respect to different colors and position with considering to Phase-Tagged triggers and spectral power, the LED's will be flickered with four shift phases aspect to phase-tagged of $\left(0^{\circ} 90^{\circ}, 180^{\circ}\right.$, and $\left.270^{\circ}\right)$ respectively, according to the setup configuration of regular pattern.

The stimuli LED's are separated into different groups of colors coordinated in center and surrounds as: RED LED's group in (respectively likewise symmetric for other two groups, BLUE LED's group arranged correspondingly, and WHITE LED's group respectively). The EEG signal was measured by single electrode placed at occipital region $(\mathrm{Oz})$ position.

The CMS (common mode sense) activates electrode and DRL (drives right leg) passive electrode, which drives average potential.

\subsection{Visual Stimuli}

Exploring a multi-color visual stimulus using a single flicker frequency respect to different colors, each visual stimulus contains a single LED. The amplitude assessment to lumens is significant large enough to SSVEP response $_{\text {evoked. }}$

Induced regular pattern with Phase-tagged triggers in each stimulus separated into groups of LED's, to cover all setup condition, in order to find out the behavioral and influences of brain activity response in all circumstances.

As shown in Fig. 2, the pattern is generated into five cycles of multi-colors stimuli rhymester on single LED which is covered an onset of (on/off _ LED) of stimulation. To provoke a regular pattern SSVEP paradigm depending on flickers besides the Phase-Tagged for each cycle according to Eq. (1):

$$
\theta_{i}=(i-1) .90^{\circ} \quad ; i=1,2, \ldots, N
$$

When flickering is shifted respect to phase angle distributed over full-phase, incremented by where taken in account in each LED stimulus will considered pulse trigger in same LED even in each cycle, implies 


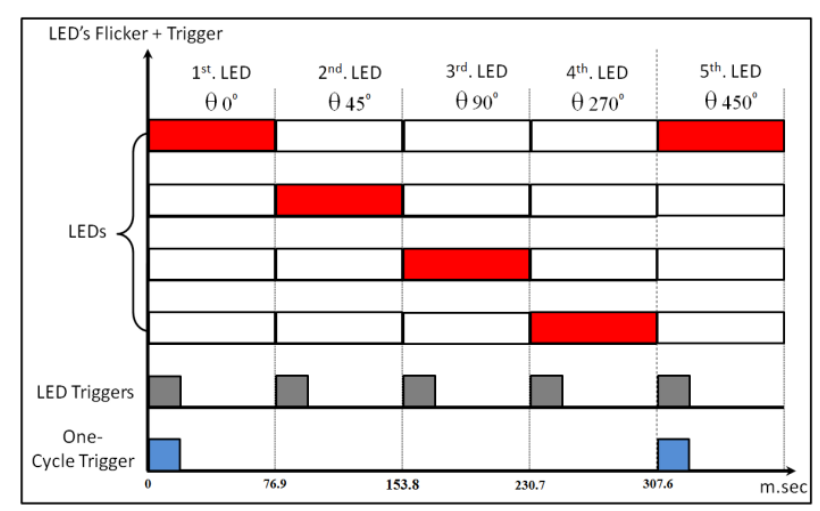

Fig. 2 A regular pattern flickering sequence on single LED for five cycles of $307.6 \mathrm{~ms}$. which include two triggers, first trigger indicate the stimulus LED, and second trigger to indicate a new cycle is begun.

controlling on the delay pulse controller depending on Eq. (2):

$$
t_{i}=\theta_{i} / 360^{\circ}
$$

$\theta_{i}$ is used as expected phase delay for evaluation performance in analysis controlling, extract $t_{i}$ to indicate the flicker LED respect to time domain. However, we can consider the $t_{i}$ which indicates the time trigger event.

The flicker frequency depends on $\theta_{i}$ as flicker sequence on all LED's, where its iteration time requested according to Eq. (3):

$$
\mathrm{T}=1 / f
$$

However, $\mathrm{T}$ is flicker cycle duration, and $f$ is the flicker frequency, indeed setting two frequencies of 6 $\mathrm{Hz}$ and $12 \mathrm{~Hz}$ surrounded by Alpha brain response range of (8 - 13) Hz.

The regular pattern generated on 4 LED's into five cycles within two type stimuli rhymester respect to Surround and Center style corresponding to the time difference between 4 LEDs of (i.e., 0, 19.225, 38.45, and 57.75) ms. based on one cycles.

Requesting from participant to gaze only on a single LED, as row stimulation, the phase delay $\theta_{i}$ in $\mathrm{LED}_{1}$ according to Eq. (1) $\left(0^{\circ}, 360^{\circ}, 720^{\circ}, 1080^{\circ}\right.$, and $\left.1440^{\circ}\right)$ respectively, however the stimuli time respect to single frequency is equivalent to the Eq. (2) as (i.e., $0,76.9,153.8,230.7$, and 307.6) ms. respectively.

Achieve two trigger events in each cycle; where the first trigger is produced in each LED Pulse-On, and a second trigger is produced with each new cycle sequence to indicate the new segment is begun, the sequence in one cycle is quite long of $307.6 \mathrm{~ms}$ Typically, using 25\% duty cycle flicking SSVEP technique achieves high SSVEP amplitude and comfortable view. Spend to kinds of stimulus flickering frequencies in low and high both bounding Alpha brain responded; where the low flicker frequency stimulus its psycho physically affects, making subjects convulsive feel visual discomfort, per contra in higher frequencies producing. Each stimulus cycle have period, contained by and, as one duty cycle represents on-time of stimulus illustrated in Fig. 2, where white-space represents the off-time and red-blocks represent a stand on-time, however the trigger respect to blue-blocks, where indicates beginning of cycle and other one indicates the LED flashing. Moreover in each step of stimuli flickering in a single square wave form are a centered with $270^{\circ}$ out of phase with neighboring squares. A four phases square waves lagging controlled by introducing a time delay $t_{i}$ of Eq. (2), creating square wave's onsets (On-Off) as one certain colors group, within events triggers.

These triggers of "phase-tagged" in cycles and each flash LED Pulse-On are assisted to extract the EEG Data in analysis as epoch-average process.

\subsection{EEG Recording and Acquisition}

EEG uses scalp electrodes connected to amplifiers and filters to detect intracranial electrical activity, which is displayed as positive or negative deflections on a line and is recorded versus time to detect different brain states.

In this study utilizes a single EEG electrode channel, were measured in Occipital Lobe at $(\mathrm{Oz})$ position accordance to the BioSemi EEG system, where encompasses the posterior portion of the human cerebral cortex and is primarily responsible for vision. Direct electrical stimulation of the Occipital Lobe produces visual sensations. 
Build in internally a BPF (band-pass-filter) within frequency range of $10-100 \mathrm{~Hz}$ in BioSemi EEG system, and a sampling rate of $2 \mathrm{kHz}$. The BioSemi replaces the "ground" electrodes which are used in conventional systems with two separate electrodes at (CMS, and DRL).

Participant 5 subjects aged between 23 and 39 years, each subject was requested to gaze at flicker LED's twice of 20s recording in both the control and the application studies, being requested to gaze at the flashing of individual groups of LED's in the formal of experiment protocol.

\subsection{Signal Analysis}

This experimental study discovers that a RED, BLUE, and WHITE colors flicker LEDs causes a changing in phase and amplitude in brain signal activity, therefore we depend on two analysis methods to distinguish the brain responses.

Firstly, an ERP method to detect the phase different in each color respect to two frequencies of $6 \mathrm{~Hz}$ and $11 \mathrm{~Hz}$ is utilized. And secondly to utilize a FFT to achieve the best power spectral in colors according to low and high frequencies. The several stimulus supports to find out the change in brain activity corresponding to the SSVEP response $_{\text {paradigms. }}$

In this study adopt offline analysis in both methods, were accumulated the EEG Data raw into epochs according to the timings of trigger signals and onsets stimulus in the flickering sequence in each group. According to the signal processing performed a LPF (low-pass-filter), HPF (high-pass-filter) to deduct unwarranted signals, and a Notch filter to remove AC-Line interference of $50 \mathrm{~Hz}$; as well a DC component is considered to remove.

Adding to process of computational parameters for each epoch was depending on the time interval of trigger windows and BL (base-line), which determine by Pre-Frame duration $250 \mathrm{~ms}$ and Post-Frame duration $500 \mathrm{~ms}$. Treating the EEG artifacts is fond of eye blinking by setting of $100 \mu \mathrm{V}$ as threshold value to reject any artifacts over the thresholds.

Finally prepare epochs averaging on all EEG Data collect basis on the timing windows of their flickering onsets to obtain SSVEP $_{\text {response }}$ to comparing the different parameters according to the brain response of LED-colors.

\section{Result}

\subsection{ERP Result}

This method for recoding and analysis of the events related to the brain potentials association with event occurs either in the external world or within the brain itself.

These potential can be provided to evaluate the activity of brain with incoming signals of around $2 \mu \mathrm{V}$ in comparison with EEG background. The analysis begins with a procedure to increase the discriminately of $\mathrm{ERP}_{\text {signal }}$ depending on the noise signal ratio. After recording all flickers-stimuli as EEG data for all sessions, we have performed an average of whole EEG segments under the same stimulus condition.

Based on our setup configuration for $\mathrm{BL}$ and Phase-tagged triggers, only the particular events average of repeated time-locked segments has been used. Then the features of EEG data as offline analysis of multi-color stimulus are extracted. This is corresponded to the $\mathrm{SSVEP}_{\text {response }}$ of brain activity recording and signal processing fundamentals of digital filters.

We configured our panel flicker-stimuli cycle length to $40 \mathrm{~s}$ respect to its $6 \mathrm{~Hz}$ flicker frequency as one cycle.

As in Figs 3 and 4, separate EEG segments for all the participant subject has been gathered and averaged. This process has been repeated for the same time-locked triggers in different colors stimulus.

The schematic in Fig. 3 represents an $\mathrm{ERP}_{\text {waveform }}$ which indicates a different quantification component. These three different curves of brain response (black, blue, and red) show the particular events occurrence 


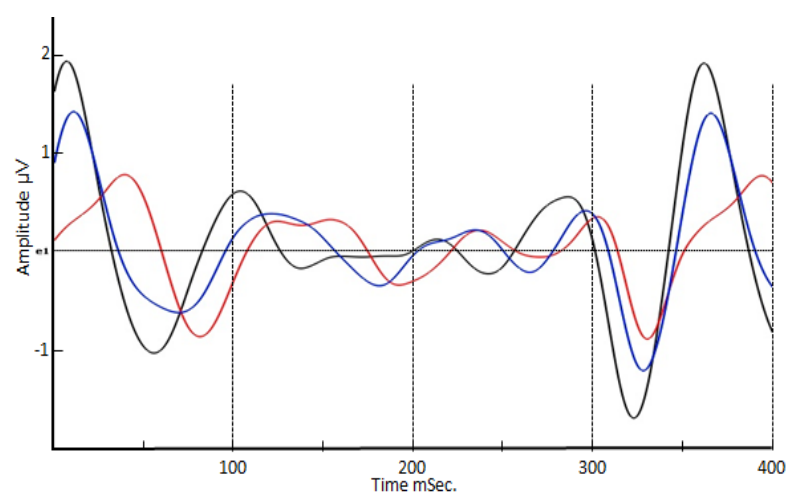

Fig. 3 Three LED's-color stimuli on fixed $6 \mathrm{~Hz}$ frequency is represented by black-red-blue respect to white-red-blue LEDs correspondingly, the amplitude in white LED color greater than other two colors, and salient different phases between three colors in negative peak ERP and in-phase on positive peak ERP.

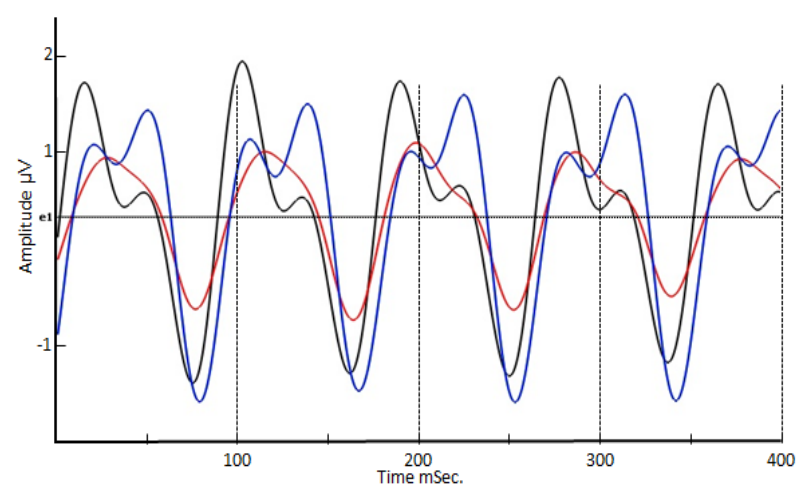

Fig. 4 Three LED's-color stimuli on fixed $12 \mathrm{~Hz}$ frequency, represent by black-red-blue respect to white-red-blue LEDs correspondingly, the amplitude in white greater than other two colors, however the frequencies are in-phase in negative ERP peak and different phase in positive peak ERP.

according to the multi-colors stimulus (i.e., white, blue, and red LEDs).

The influence of the positive and the negative peaks which are observed in figures are the different ERP brain responses for each color stimulus respect to

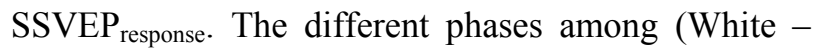
Red $=27 \mathrm{~ms})$; (Blue - White $=16 \mathrm{~ms})$ and $($ Red Blue $=15 \mathrm{~ms}$ ) are shown in figure 3 . However, we can observe the amplitudes of a white stimulus greater than other two colors.

In the same scenario of multi-colors stimuli, but in $12 \mathrm{~Hz}$ flicker frequency, Fig. 4 illustrates amplitude in white and blue stimulus greater than a red stimulus. However, the white stimulus amplitude is less than previous flicker frequency. And the negative pecks in ERP analysis look like in-phase.

In other hands, discriminate delay phase in positive peak ERP, but conversely the phase delay at $6 \mathrm{~Hz}$ flicker frequency on negative peak ERP.

\subsection{FFT Result}

The basic approach to signal analysis is to get proper information from the signal by applying the FFT algorithm.

The spectral analysis of a signal involves decomposition into frequency components domain. In other words, the original signal can be separated into its sub-spectral components by using spectral analysis methods of FFT. Apply the digital filter after accumulated, then averaged of EEG data in all sessions. Implement a LPF with $40 \mathrm{~Hz}$ cutoff frequency and HPF in $4 \mathrm{~Hz}$ as cutoff frequency.

Referring to regular SSVEP paradigms of stimuli based on BCI, the configuration conditions of experiment are steady in all sessions. The Figs. 5-10, are illustrating the result of brain activity in RED, BLUE, and WHITE LEDs stimulus, where they are using only flicker frequencies in $6 \mathrm{~Hz}$ and $12 \mathrm{~Hz}$. The Figs. 5-10 are shown different power spectrum respect

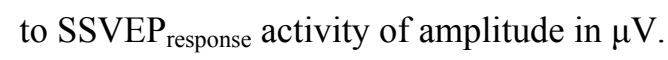

Comparing our previous ERP results, we found they have approximately same responded activities level in both frequencies. However, obviously WHITE Stimuli has a greater amplitude compare with the two other stimulus (RED and BLUE).

\section{Conclusion}

In this paper, we present the results of experimental study demonstrated a successfully low-cost prototype SSVEP based on BCI system, which constructed a handmade stimulation panel to evoke participants. The motivation of this study is to develop and define a visual stimulus depends on SSVEPresponse based on BCI systems to relieve the visual tiredness. Through the experiment, we preferred the regular flicker paradigms 


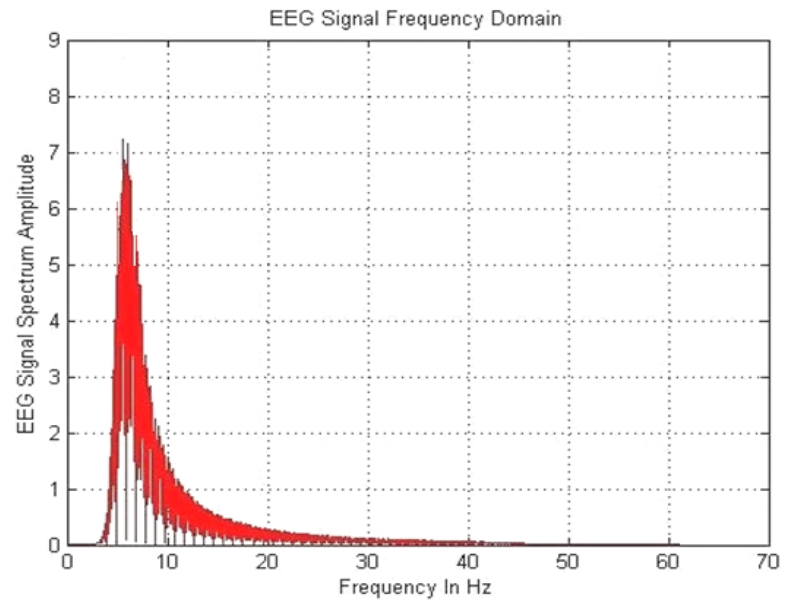

Fig. 5 The brain activity of SSVEP response $_{\text {in RED stimuli }}$ at low flicker frequency of $6 \mathrm{~Hz}$ with amplitude of $7.2 \mu \mathrm{V}$.

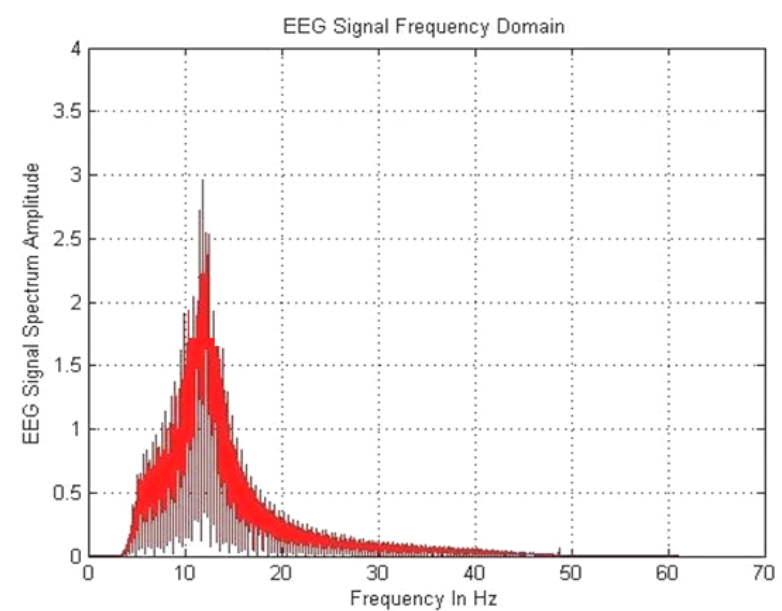

Fig. 6 The brain activity of SSVEP $P_{\text {response }}$ in RED stimuli at high flicker frequency of $12 \mathrm{~Hz}$ with amplitude of $2.9 \mu \mathrm{V}$.

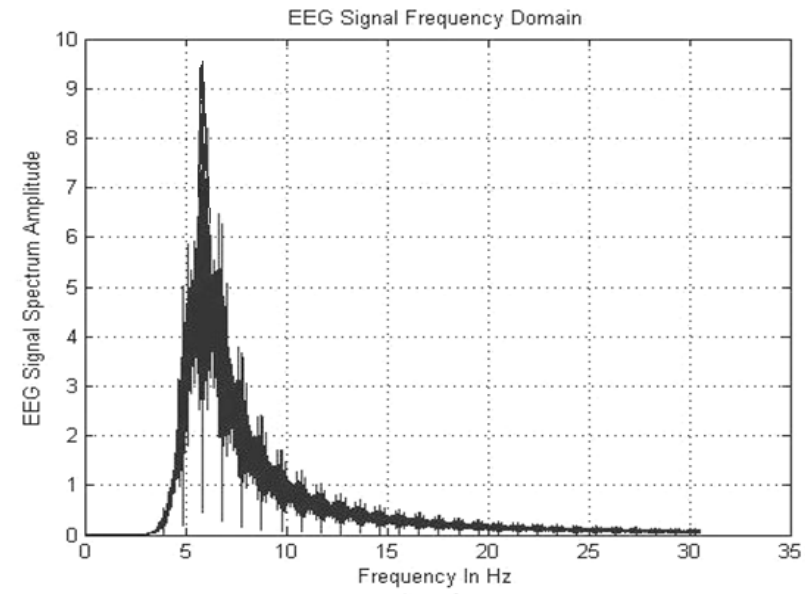

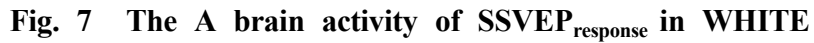
stimuli at low flicker frequency of $6 \mathrm{~Hz}$ with amplitude of $9.6 \mu \mathrm{V}$.

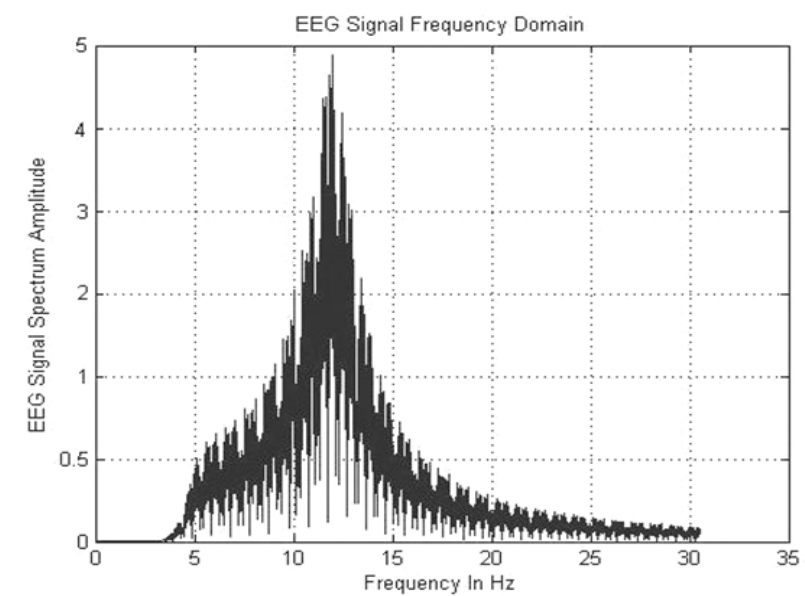

Fig. 8 The brain activity of SSVEP response $_{\text {in WHITE stimuli }}$ at high flicker frequency of $12 \mathrm{~Hz}$ with amplitude of $4.9 \mu \mathrm{V}$.

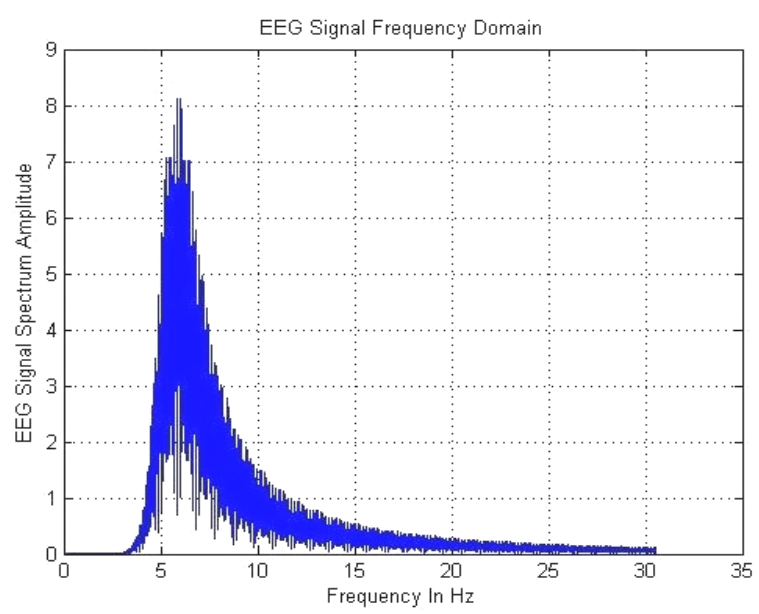

Fig. 9 The brain activity of SSVEP response $_{\text {in BLUE stimuli at }}$ low flicker frequency of $6 \mathrm{~Hz}$ with amplitude of $8.2 \mu \mathrm{V}$.

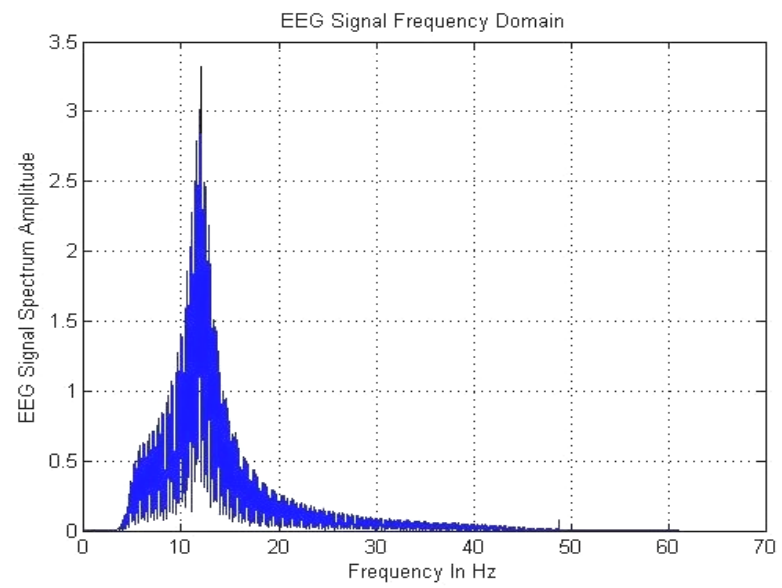

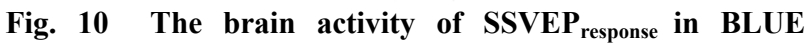
stimuli at high flicker frequency of $12 \mathrm{~Hz}$ with amplitude of $3.3 \mu \mathrm{V}$. 
to observe the brain response. The experiment results are obtained on two analysis methods; ERP in which reveal the phase shifted behavioral in colors stimulus and the FFT to detect the maximum amplitude power in each colors. We have found the different influence in SSVEPresponse respect to brain activity performance such as: The SSVEPs elicited by the white-color has a highest brain activity compared with Red and Blue colors, thus lead as the white color is still the best of encoding, and achieves a highest accuracy. The other one is, Blue colors elicit from SSVEPs high phase-shift with highest frequency, in other hand the Red color in low frequency. Multi-colors could be helpful in new applications as a supporter to handicap peoples for more attractive external environment. This will make a more objective comparison among $\mathrm{BCI}$ systems with single and multiple colors. A multi-colors paradigm will introduce a different protocol for different applications. Finally this gave us the advantages to exploit this method as a future application work in SSVEP based on BCI system fields since it is adapted, optimized, and configured with support of FPGA as online experiments.

\section{Acknowledgments}

Support for this work, by University of Rostock, was provided by Computer Science Department. And the authors would like to thanks in advance for all participated persons as subjects in this work.

\section{References}

[1] Vialatte, F.-B., Maurice, M., Dauwels, J., and Cichocki, A. 2010. "Steady-State Visually Evoked Potentials: Focus on Essential Paradigms and Future Perspectives." Progress in Neurobiology90 (4): 418-38.

[2] Garcia, G. 2008. "High Frequency SSVEPs for BCI
Applications." Philips Research Europe.-High Tech Campus 34, 5656 AE, Eindhoven - The Netherlands

[3] Punsawad, Y., and Wongsawat, Y. 2012. "Motion Visual Stimulus for SSVEP Based BCI System." In Proceedings of the Annual International Conference of the IEEE Engineering in Medicine and Biology Society (EMBC), 3837-40.

[4] Yue, L., Xiao, J., Teng, C., Feng, W., Un, M. P., and Pui-In, M. 2012. "Implementation of SSVEP Based BCI with Emotiv EPOC." In Proceedings of the Virtual Environments of Human-Computer Interfaces and Measurement Systems (VECIMS), 34-7.

[5] Falzon, O., and Camilleri, K. P. 2013. "Multi-colour Stimuli to Improve Information Transfer Rates in SSVEP-Based Brain-Computer Interfaces.” Presented at the IEEE EMBS Conference on Neural Engineering, San Diego, California.

[6] Mouli, S., Palaniappan, R., Sillitoe, I. P., and Gan, J. Q. 2013. "Performance Analysis of Multi-frequency SSVEP-BCI Using Clear and Frosted Colour LED Stimuli”. In Proceedings of the 2013 IEEE 13th International Conference on Bioinformatics and Bioengineering,1-4

[7] Zhu, D., Bieger, J., Molina, G. G., andAarts,R. M. 2010. "A Survey of Stimulation Methods Used in SSVEP-Based BCIs." Computational Intelligence and Neuroscience $2010: 1$.

[8] Falzon, O., Camilleri, K., and Muscat, J. 2012. "Complex-Valued Spatial Filters for SSVEP-Based BCIs with Phase Coding." IEEE Transactions onBiomedical Engineering 59 (9): 2486-95.

[9] Cao, T., Wan, F., Mak, P. U., Mak, P.-I., Vai, M. I., and Hu, Y. 2012. "Flashing Color on the Performance of SSVEP-Based Brain-Computer Interfaces." In Proceedings of the 2012 34th Annual International Conference of the IEEE Engineering in Medicine and Biology Society, 1819-22.

[10] Cheng, M., Gao, X., Gao, S., and Xu, D. 2001. "Multiple Color Stimulus Induced Steady State Visual Evoked Potentials." In Proceedings of the 23rd Annual International Conference of the IEEE, 2 : 1012-14.

[11] Cao, T., Wan, F., Mak, P. U., Mak, Pui-I., Vai, M. I.,and Hu, Y. 2012. "Flashing Color on the Performance of SSVEP-Based Brain-Computer Interfaces." Presented at the 34th Annual International Conference of the IEEE EMBS, San Diego, California, USA. 